\title{
Anticancer effects of the microbiota: how the microbiome shapes the development of IL-9-producing T cells
}

IL-9-producing T cells can harbour potent anti-cancer functions. In this issue of the British Journal of Cancer, Almeida et al. found that the host microbiota enhances in vivo T cell-derived secretion of IL-9, thereby limiting cancer outgrowth.

British Journal of Cancer (2020) 123:497-498; https://doi.org/10.1038/s41416-020-0936-1

\section{MAIN}

While the ability of transforming growth factor (TGF)- $\beta$ and interleukin (IL)-4 to induce IL-9 secretion from T cells dates back to $1994,{ }^{1}$ it was only in 2008 that IL-9-secreting CD4 T cells ( $T_{H} 9$ cells) were characterised. ${ }^{2}$ Beyond their high secretion of IL-9, $T_{H} 9$ cells secrete IL-10 and IL-21 and are highly pro-inflammatory in vivo. Subsequent studies have shown that $\mathrm{T}_{\mathrm{H}} 9$ cells contribute to antitumour immunity, notably in the context of melanoma. Like $\mathrm{T}_{\mathrm{H}} 9$ cells, IL-9-producing CD8 $\mathrm{T}$ cells, termed Tc9 cells, were identified as potent effector $T$ cells in vivo. The adoptive transfer of IL-9-producing $T$ cells ( $T_{H}$ 9 or Tc9) cells into tumour-bearing mice leads to cancer elimination in vivo through direct anticancer effects as well as induction of anticancer immune responses. ${ }^{3-5}$ The relevance of these findings has been extended to humans, as underscored by the better clinical prognosis of cancer patients with high IL-9-producing cell infiltration. ${ }^{6}$ The environmental factors responsible for the induction of IL-9-producing $T$ cells in vivo remain elusive.

In this issue of British Journal of Cancer, Almeida et al. uncovered a major contribution of the microbiota in inducing IL-9-producing T cells in mice. ${ }^{7}$ By interrogating the frequency of IL-9-producing T cells in the colonic lamina propria of germ-free or control mice, they found that the former featured a reduced frequency of both $T_{H} 9$ and Tc9 cells. Elimination of the microbiota in control mice with antibiotics also led to a reduction in their frequency of colonic $\mathrm{T}_{\mathrm{H}} 9$ and Tc9 cells. ${ }^{7}$ TGF- $\beta$ and IL- 4 were shown to contribute to $T_{H} 9$ and Tc9 cell differentiation. ${ }^{2,3}$ Interestingly, Almeida et al. found that antibiotics reduced the expression of TGF- $\beta$ and IL- 4 in the colonic tissue, suggesting that the microbiota supports the generation of IL-9-producing T cells through the induction of these two cytokines (Fig. 1). ${ }^{7}$ Whether the microbiota affects the functions of IL-9-producing $T$ cells was then investigated in the setting of cancer. In the B16F10 mouse melanoma model, the authors found that mice treated with antibiotics featured enhanced tumour progression, which was associated with a lower frequency of IL-9-producing T cells at the tumour site. This could be reversed by the addition of recombinant IL-9 protein, suggesting that IL-9 is the main effector cytokine driving the anticancer effects in the authors' model (Fig. 1). ${ }^{7}$ IL-9 can be secreted by other cell types such as mast cells, invariant natural killer (NK) T cells and type 2 innate lymphoid cells. ${ }^{8}$ However, similar levels of non-T cells secreting IL9 were found in tumour-bearing mice irrespective of antibiotic treatment, suggesting that the effects of microbiota are due to $\mathrm{T}$ cell-derived IL-9.'

Proinflammatory factors have been reported to profoundly affect $T$ differentiation. IL-1 $\beta$ enhances both the differentiation and effector functions of $T_{H} 17$ and $T_{H} 9$ cells. Likewise, the observations of Almeida et al. that the microbiota supports the generation of IL-9-producing $T$ cells are reminiscent of the known ability of bacteria to affect $T_{H} 17$ cell differentiation. ${ }^{9}$ Whether a similar microbial environment promotes IL-17- and IL-9-producing $\mathrm{T}$ cell generation was not addressed by Almeida et al. Likewise, how the microbiota supports the anticancer functions of IL-9producing $T$ cells remains unclear. While IL-9 has been proposed to contribute to CD8 T cell recruitment, the authors found that recombinant IL-9 is not affecting the frequency of CD8 T cell infiltration in the lungs. ${ }^{7}$ However, whether IL-9 administration affects the activation status of innate immune cells such as mast and NK cells, which were shown to contribute to B16F10 tumour elimination, was not investigated (Fig. 1).

While IL-9 was originally shown to promote T cell growth, subsequent studies have demonstrated the pleiotropic functions of IL-9. ${ }^{8}$ One of the notable features of IL-9 is the ability to affect tissue barrier function. In this regard, Renga et al. uncovered that inflammatory dysbiosis can occur in the absence of IL- $9{ }^{10}$ raising the hypothesis that IL-9 affects the microbiota. Is there a crossregulation between the microbiota and IL-9 in cancer? While the

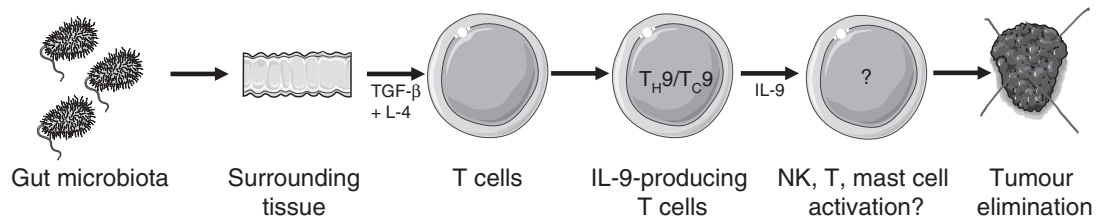

Fig. 1 The microbiota harnesses the anticancer functions of IL-9-producing $\mathbf{T}$ cells. In tumour-bearing mice, the microbiota drives the induction of IL-9-producing T cells, possibly through favouring TGF- $\beta$ and IL-4 expression from surrounding tissues. IL-9-producing T cells then exert anticancer effects either by directly eliminating tumour cells or by mobilising additional immune effectors. 
answer to this question remains unclear at this stage, the study of Almeida et al. ${ }^{7}$ provides evidence that this is a possibility worth investigating.

Although the results of Almeida et al. $^{7}$ in the B16F10 mouse melanoma tumour model still need to be extended to other tumour models and to a human setting, they are raising exciting hypotheses that reach beyond the modulations of the anticancer functions of IL9-producing $T$ cells. The microbiota indeed not only affects the induction of anticancer immune responses, but was also recently shown to determine the response to anti-programmed cell death protein 1 (anti-PD-1) therapy in multiple cancer types. ${ }^{11,12}$ Likewise, the frequency of circulating $T_{H} 9$ cells has been proposed to be associated with the response to anti-PD-1 therapy in humans. ${ }^{13}$ These observations, along with the results of Almeida et al., ${ }^{7}$ prompt for future studies investigating whether the induction of $T$ cellderived IL-9 promoted by the microbiota contributes to the anticancer response observed following anti-PD-1 treatment. In summary, Almeida et al. ${ }^{7}$ showed that the development and anticancer functions of IL-9-producing cells can be affected by the microbiota. These results contribute to a better understanding of $T$ cell biology and set bases for novel studies that could possibly unravel novel biomarkers of cancer immunotherapy treatments.

\section{AUTHOR CONTRIBUTIONS}

L.A. wrote and approved the final paper.

\section{ADDITIONAL INFORMATION}

Ethics approval and consent to participate Not applicable.

Data availability Not applicable.

Competing interests The author declares no competing interests.

Funding information L.A. is funded by grants from the Fondation de France, the Conseil Régional de Bourgogne and FEDER, the Agence Nationale de la Recherche [ANR-11-LABX-0021], the Acteria Foundation and the European Research Council (ERC) (grant agreement No. 677251).

Note This work is published under the standard license to publish agreement. After 12 months the work will become freely available and the license terms will switch to a Creative Commons Attribution 4.0 International (CC BY 4.0).

Publisher's note Springer Nature remains neutral with regard to jurisdictional claims in published maps and institutional affiliations.
Lionel Apetoh ${ }^{1,2}$

'INSERM, U1231, Dijon, France and ${ }^{2}$ Faculté de Médecine, Université de Bourgogne Franche Comté, Dijon, France Correspondence: Lionel Apetoh (lionel.apetoh@inserm.fr)

\section{REFERENCES}

1. Schmitt, E., Germann, T., Goedert, S., Hoehn, P., Huels, C., Koelsch, S. et al. IL-9 production of naive CD4+ T cells depends on IL-2, is synergistically enhanced by a combination of TGF-beta and IL-4, and is inhibited by IFN-gamma. J. Immunol. 153, 3989-3996 (1994).

2. Dardalhon, V., Awasthi, A., Kwon, H., Galileos, G., Gao, W., Sobel, R. A. et al. IL-4 inhibits TGF-beta-induced Foxp3+ T cells and, together with TGF-beta, generates IL-9+ IL-10+ Foxp3(-) effector T cells. Nat. Immunol. 9, 1347-1355 (2008).

3. Lu, Y., Hong, B., Li, H., Zheng, Y., Zhang, M., Wang, S. et al. Tumor-specific IL-9producing CD8+ Tc9 cells are superior effector than type-I cytotoxic Tc1 cells for adoptive immunotherapy of cancers. Proc. Natl Acad. Sci. USA 111, 2265-2270 (2014).

4. Vegran, F., Berger, H., Boidot, R., Mignot, G., Bruchard, M., Dosset, M. et al. The transcription factor IRF1 dictates the IL-21-dependent anticancer functions of TH9 cells. Nat. Immunol. 15, 758-766 (2014).

5. Lu, Y., Wang, Q., Xue, G., Bi, E., Ma, X., Wang, A. et al. Th9 cells represent a unique subset of CD4(+) T cells endowed with the ability to eradicate advanced tumors. Cancer Cell 33, 1048-1060 (2018).

6. Wang, J., Sun, M., Zhao, H., Huang, Y., Li, D., Mao, D. et al. IL-9 exerts antitumor effects in colon cancer and transforms the tumor microenvironment in vivo. Technol. Cancer Res. Treat. 18, 1533033819857737 (2019).

7. Almeida, R. R., de Souza Vieira, R., Castoldi, A., Terra, F. F., Melo, A. C. L., Canesso, M. C. C. et al. Host dysbiosis negatively impacts IL-9-producing T-cell differentiation and antitumor immunity. Br. J. Cancer https://doi.org/10.1038/s41416020-0915-6 (2020).

8. Kaplan, M. H., Hufford, M. M. \& Olson, M. R. The development and in vivo function of T helper 9 cells. Nat. Rev. Immunol. 15, 295-307 (2015).

9. Ivanov, I. I., Atarashi, K., Manel, N., Brodie, E. L., Shima, T., Karaoz, U. et al. Induction of intestinal Th17 cells by segmented filamentous bacteria. Cell 139, 485-498 (2009).

10. Renga, G., Moretti, S., Oikonomou, V., Borghi, M., Zelante, T., Paolicelli, G. et al. IL-9 and mast cells are key players of Candida albicans commensalism and pathogenesis in the gut. Cell Rep. 23, 1767-1778 (2018).

11. Gopalakrishnan, V., Spencer, C. N., Nezi, L., Reuben, A., Andrews, M. C., Karpinets, T. V. et al. Gut microbiome modulates response to anti-PD-1 immunotherapy in melanoma patients. Science 359, 97-103 (2018).

12. Routy, B., Le Chatelier, E., Derosa, L., Duong, C. P. M., Alou, M. T., Daillere, R. et al. Gut microbiome influences efficacy of PD-1-based immunotherapy against epithelial tumors. Science 359, 91-97 (2018).

13. Nonomura, Y., Otsuka, A., Nakashima, C., Seidel, J. A., Kitoh, A., Dainichi, T. et al. Peripheral blood Th9 cells are a possible pharmacodynamic biomarker of nivolumab treatment efficacy in metastatic melanoma patients. Oncoimmunology $\mathbf{5}$, e1248327 (2016). 